Tôhoku Math. Journ.

31 (1979), 195-205.

\title{
RANKIN'S METHOD IN THE CASE OF LEVEL $4 q$ AND ITS APPLICATION TO THE DOI-NAGANUMA LIFTING
}

\author{
HISASHI KOJIMA
}

(Received May 19, 1978, revised July 31, 1978)

Introduction. Let $S_{k}\left(\Gamma_{0}(N), \chi\right)$ be the space of integral cusp forms of Neben-type $\chi$ and of weight $k$ with respect to $\Gamma_{0}(N)$. We associate with a cusp $p$ of $\Gamma_{0}(N)$ a matrix $\alpha_{p}\left(\in S L_{2}(R)\right)$ such that $\alpha_{p}(\infty)=p$. Assume that $k$ is an even positive integer. Then every $f \in S_{k}\left(\Gamma_{0}(N), \chi\right)$ has the Fourier expansion $\left(f \mid\left[\alpha_{p}\right]_{k}\right)(z)=\sum_{n=1}^{\infty} a_{n}^{(p)} e^{2 \pi i n z / \beta}$ at $p$ for some $\beta>0$. The numbers $\left\{a_{n}^{(p)}\right\}_{n=1}^{\infty}$ are called the Fourier coefficients of $f$ at $p$.

When we apply Rankin's method to the Dirichlet series corresponding to an automorphic form in $S_{k}\left(\Gamma_{0}(N), \chi\right)$, certain explicit relations between the Fourier coefficients at all cusps are needed. In this paper, we deal with the problem: Given the coefficients at one cusp, can all coefficients at other cusps be determined? Recently, by using the $W$ matrix in Atkin-Lehner [2] and Hecke operators, Asai [1] solved the problem positively in the case where $N$ is square-free. If $N$ is not square-free, we cannot immediately apply his argument.

In $\S 1$, by a different method, we give an affirmative answer to the above problem in the case $N=4 q$ with $q$ prime. $\S 2$ and $\S 3$ are preparatory sections, where we describe certain properties of Eisenstein-Epstein functions and Maass' theta functions and, in the last section, we give an application of the result in $\S 1$ to the Doi-Naganuma lifting in the case of $\boldsymbol{Q}(\sqrt{4 q})$ with a prime $q \equiv 3(\bmod 4)$. The basic references for this subject are Asai [1], Doi-Naganuma [3], Naganuma [5], Shimura [7] and Zagier [8].

1. Fourier coefficients at various cusps. Throughout this paper, we use the following notations. Let $N$ be a positive integer and let $\chi$ be a Dirichlet character modulo $N$. Put

$$
\Gamma_{0}(N)=\left\{\left(\begin{array}{ll}
a & b \\
c & d
\end{array}\right) \in S L_{2}(Z) \mid c \equiv 0 \bmod N\right\} .
$$

We let $\mathscr{B}$ denote the complex upper half plane. Assume that $f$ is a holomorphic function on $\mathfrak{S}$. Put $\left(f \mid[\sigma]_{k}\right)(z)=(\operatorname{det} \sigma)^{k / 2}(c z+d)^{-k} f(\sigma(z))$ for $\sigma=\left(\begin{array}{ll}a & b \\ c & d\end{array}\right) \in G L_{2}^{+}(\boldsymbol{R})$. We denote by $S_{k}\left(\Gamma_{0}(N), \chi\right)$ and by $S_{k}^{0}\left(\Gamma_{0}(N), \chi\right)$ 
the space of integral cusp forms of Neben-type $\chi$ and of weight $k$ with respect to $\Gamma_{0}(N)$ and the subspace of new forms in the sense of AtkinLehner, respectively. For a prime $p$, we define the Hecke operator $T(p, \chi)$ on $S_{k}\left(\Gamma_{0}(N), \chi\right)$ by

$$
f \mid T(p, \chi)=p^{k / 2-1}\left\{\chi(p) f\left|\left(\begin{array}{ll}
p & 0 \\
0 & 1
\end{array}\right)_{k}+\sum_{j=0}^{p-1} f\right|\left(\begin{array}{ll}
1 & j \\
0 & p
\end{array}\right)_{k}\right\} .
$$

Let $q \equiv 3(\bmod 4)$ be a prime and take $N$ to be $4 q$. For a divisor $M$ of $4 q$, we define the matrix $\alpha_{M}$ by

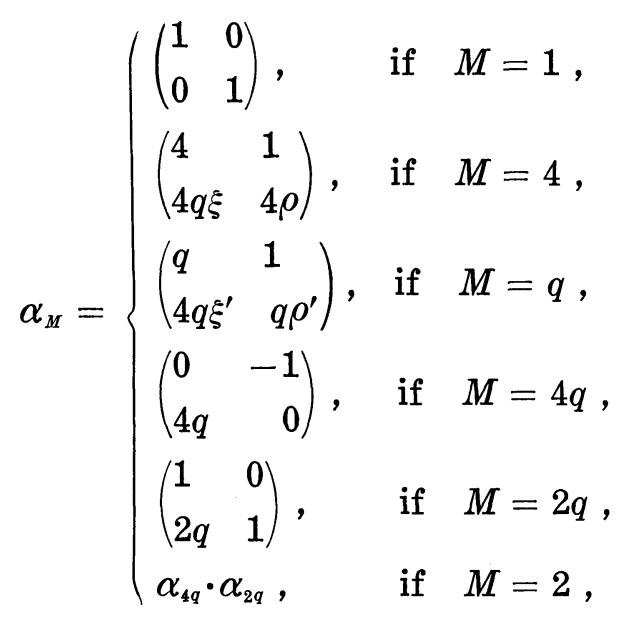

where $\xi, \rho, \xi^{\prime}$ and $\rho^{\prime} \in Z, 4 \rho-q \xi=1$ and $q \rho^{\prime}-4 \xi^{\prime}=1$. Here we note that $\alpha_{M}$ normalizes $\Gamma_{0}(4 q)$.

Let $f(z)$ be a new form of $S_{k}\left(\Gamma_{0}(4 q),\left(\frac{4 q}{*}\right)\right)$ with the Fourier expansion $f(z)=\sum_{n=1}^{\infty} a_{n} e^{2 \pi i n z}\left(a_{1}=1\right)$, so that $f \mid T\left(p,\left(\frac{4 q}{*}\right)\right)=a_{p} f$ for each prime $p$. We now determine $f \mid\left[\alpha_{M}\right]_{k}$ in an explicit form. First, we recall some well-known facts (cf. [4]).

Let $N$ be a positive integer and let $q$ be a prime such that $q \mid N$. We easily obtain the natural isomorphism $(\boldsymbol{Z} / N \boldsymbol{Z})^{\times} \cong \Pi_{q \mid N}\left(\boldsymbol{Z} / N_{q} \boldsymbol{Z}\right)^{\times}$, where $N_{q}$ is the $q$-factor of $N$. We denote by $\chi_{q}$ the induced character modulo $N_{q}$. Define $\gamma_{q}\left(\in S L_{2}(\boldsymbol{Z})\right)$ and $\gamma_{q}^{\prime}\left(\in S L_{2}(\boldsymbol{Z})\right)$ by

$(*) \quad \gamma_{q} \equiv\left\{\begin{array}{ll}\left(\begin{array}{rr}0 & -1 \\ 1 & 0\end{array}\right) & \left(\bmod N_{q}^{2}\right), \\ \left(\begin{array}{rr}1 & 0 \\ 0 & 1\end{array}\right) & \left(\bmod \left(N / N_{q}\right)^{2}\right)\end{array} \quad\right.$ and $\quad \gamma_{q}^{\prime} \equiv\left\{\begin{array}{ll}\left(\begin{array}{rr}1 & 0 \\ 0 & 1\end{array}\right)\left(\bmod N_{q}^{2}\right), \\ 0 & -1 \\ 1 & 0\end{array}\right)\left(\bmod \left(N / N_{q}\right)^{2}\right)$ and we put 


$$
\eta_{q}=\gamma_{q}\left(\begin{array}{ll}
N_{q} & 0 \\
0 & 1
\end{array}\right) \quad \text { and } \quad \eta_{q}^{\prime}=\gamma_{q}^{\prime}\left(\begin{array}{ll}
N / N_{q} & 0 \\
0 & 1
\end{array}\right) \text {. }
$$

The following theorem is well-known (cf. [4]).

THEOREM. (i) There exist two isomorphisms

$$
S_{k}^{0}\left(\Gamma_{0}(N), \chi\right) \stackrel{\left[\eta_{q}\right]_{k}}{\longrightarrow} S_{k}^{\circ}\left(\Gamma_{0}(N),\left(\prod_{p \neq q} \chi_{p}\right) \bar{\chi}_{q}\right)
$$

and

$$
S_{k}^{0}\left(\Gamma_{0}(N), \chi\right) \stackrel{\left[\eta_{q}^{\prime}\right]_{k}}{\longrightarrow} S_{k}^{0}\left(\Gamma_{0}(N),\left(\prod_{p \neq q} \bar{\chi}_{p}\right) \chi_{q}\right)
$$

(ii) Let $f$ be an element in $S_{k}\left(\Gamma_{0}(N), \chi\right)$. Then $f \mid\left[\eta_{q} \eta_{q}^{\prime}\right]_{k}=$ $\bar{\chi}_{q}^{\prime}\left(N_{q}\right) f \mid\left(\begin{array}{rr}0 & -1 \\ N & 0\end{array}\right)_{k}$, where $\chi_{q}^{\prime}=\prod_{p \neq q} \chi_{p}$.

(iii) Suppose that $f$ is a primitive form in the sense of AtkinLehner and $\chi_{q}$ is a primitive character. Then $g_{q}$ is also a primitive form and $f \mid\left[\eta_{q}\right]_{k}=\chi_{q}(-1) \bar{a}_{q}^{e} w\left(\chi_{q}\right) q^{-(e k / 2)} g_{q}$, where $w\left(\chi_{q}\right)=\sum_{a=0}^{N q-1} \chi_{q}(a) e^{2 \pi i a / N_{q}}$, $N_{q}=q^{e}$ and $g_{q}(z)=\sum_{n=1}^{\infty} b_{n}^{(q)} e^{2 \pi i n z}$ with

$$
b_{p}^{(q)}=\left\{\begin{array}{lll}
\bar{\chi}_{q}(p) a_{p} & \text { if } & p \neq q \\
\chi_{q}^{\prime}(p) \bar{a}_{p} & \text { if } & p=q
\end{array}\right.
$$

for every prime p. Moreover $\left|a_{q}\right|^{2}=q^{k-1}$.

Now we can prove the following:

Proposition 1.1. Let $f(z)$ be a primitive form of $S_{k}\left(\Gamma_{0}(4 q),\left(\frac{4 q}{*}\right)\right)$ with the Fourier expansion $f(z)=\sum_{n=1}^{\infty} a_{n} e^{2 \pi i n z}$. Then

$$
f \mid\left[\alpha_{4 q}\right]_{k}=\bar{a}_{4 q}(2 \sqrt{q})^{-(k-1)} f_{\rho},
$$

where $f_{\rho}(z)=\sum_{n=1}^{\infty} \bar{a}_{n} e^{2 \pi i n z}$.

Proof. First we observe that $N=4 q$ and $\chi=\left(\frac{4 q}{*}\right)$. Let $\gamma_{2}, \gamma_{q}, \eta_{2}$, and $\eta_{q}$ be matrices satisfying the conditions $(*),(* *)$ and let

$$
\gamma_{2}^{\prime}=\gamma_{q}, \eta_{2}^{\prime}=\gamma_{2}^{\prime}\left(\begin{array}{ll}
N / N_{2} & 0 \\
0 & 1
\end{array}\right)=\gamma_{2}^{\prime}\left(\begin{array}{ll}
q & 0 \\
0 & 1
\end{array}\right) \text {. }
$$

Then we see that $\gamma_{2}^{\prime}$ and $\eta_{2}^{\prime}$ satisfy the conditions $(*),(* *)$ of the above theorem again.

By (ii), we see that $f\left|\left[\alpha_{4 q}\right]_{k}=f\right|\left[\eta_{2} \eta_{2}^{\prime}\right]_{k}=f \mid\left[\eta_{2} \eta_{q}\right]_{k}$. Therefore, it is sufficient to determine $f\left|\left[\eta_{2}\right]_{k}, f\right|\left[\eta_{2}\right]_{k} \mid\left[\eta_{q}\right]_{k}$. By (iii), we have $f \mid\left[\eta_{2}\right]_{k}=$ $\bar{a}_{4}(-2 i) 2^{-k} \widetilde{g}$, where $\widetilde{g}(z)=\sum_{n=1}^{\infty} a_{n}^{(1)} e^{2 \pi i n z}$ with 


$$
a_{p}^{(1)}=\left\{\begin{array}{lll}
\left(\frac{-4}{p}\right) a_{p} & \text { if } & p \neq 2, \\
\left(\frac{p}{q}\right) \bar{a}_{p} & \text { if } & p=2 .
\end{array}\right.
$$

It should be noted that $\widetilde{g}$ is a primitive form. We have

$$
f\left|\left[\eta_{2} \eta_{q}\right]_{k}=\bar{a}_{4}(-2 i) 2^{-k} \widetilde{g}\right|\left[\eta_{q}\right]_{k}
$$

and

$$
\widetilde{g} \mid\left[\eta_{q}\right]_{k}=\bar{a}_{q}(\sqrt{q i}) q^{-k / 2} \tilde{h},
$$

where $\tilde{h}=\sum_{n=1}^{\infty} a_{n}^{(2)} e^{2 \pi i n z}$ with

$$
a_{p}^{(2)}=\left\{\begin{array}{lll}
\left(\frac{p}{q}\right) a_{p}^{(1)} & \text { if } & p \neq q, \\
\left(\frac{-4}{p}\right) \overline{a_{p}^{(1)}} & \text { if } & p=q,
\end{array}\right.
$$

hence $a_{n}^{(2)}=\bar{a}_{n}$. The proof is complete.

Proposition 1.2. Under the same conditions as in Proposition 1.1, we have $f \mid\left[\alpha_{2 q}\right]_{k}=2^{1-k} \bar{a}_{2} \sum_{n=1}^{\infty} a_{2 n-1} e^{\pi i(2 n-1) z}$.

Proof. By Proposition 1.1, we see that

$$
f=\bar{a}_{4 q} \sqrt{4 q}^{-(k-1)} f_{\rho} \mid\left[\alpha_{4 q}\right]_{k}
$$

and

$$
f\left|\left[\alpha_{2 q}\right]_{k}=\bar{a}_{4 q} \sqrt{4 q}^{-(k-1)} f_{\rho}\right|\left[\alpha_{4 q} \alpha_{2 q}\right]_{k}
$$

Note that

$$
\alpha_{4 q} \alpha_{2 q}=\left(\begin{array}{rr}
-2 q & -1 \\
4 q & 0
\end{array}\right) \text {. }
$$

Then we have $f_{\rho} \mid\left[\alpha_{4 q} \alpha_{2 q}\right]_{k}(z)=f_{\rho}(-1 / 2-1 / 4 q z)(4 q)^{-k / 2} z^{-k}$. We put $f_{\rho}(z)=$ $f_{\rho}^{(1)}(z)+f_{\rho}^{(2)}(z)$, where $f_{\rho}^{(1)}(z)=\sum_{n=0}^{\infty} \bar{a}_{2 n+1} e^{2 \pi i(2 n+1) z}$ and $f_{\rho}^{(2)}(z)=\sum_{n=1}^{\infty} \bar{a}_{2 n} e^{2 \pi i(2 n) z}$. Since $f$ is an eigenfunction of $T\left(2,\left(\frac{4 q}{*}\right)\right)$, we see that

$$
f_{\rho}^{(2)}(z)=\sum_{n=1}^{\infty} \bar{a}_{2 n} e^{2 \pi i(2 n z)}=\bar{a}_{2} \sum_{n=1}^{\infty} \bar{a}_{n} e^{2 \pi i(2 n z)}=\bar{a}_{2} f_{\rho}(2 z) .
$$

So we have

$$
\begin{aligned}
f_{\rho}(- & 1 / 2-1 / 4 q z)(4 q)^{-k / 2} z^{-k} \\
& =\left\{f_{\rho}^{(1)}(-1 / 2-1 / 4 q z)+f_{\rho}^{(2)}(-1 / 2-1 / 4 q z)\right\}(4 q)^{-k / 2} z^{-k} \\
& =\left\{-f_{\rho}^{(1)}(-1 / 4 q z)+\bar{a}_{2} f_{\rho}(-1 / 2 q z)\right\}(4 q)^{-k / 2} z^{-k}
\end{aligned}
$$




$$
\begin{aligned}
& =\left\{2 \bar{a}_{2} f_{\rho}(-1 / 2 q z)-f_{\rho}(-1 / 4 q z)\right\}(4 q)^{-k / 2} z^{-k} \\
& =2^{1-k} \bar{a}_{2} f_{\rho}\left|\left[\alpha_{4 q}\right]_{k}(z / 2)-f_{\rho}\right|\left[\alpha_{4 q}\right]_{k}(z) .
\end{aligned}
$$

Hence $f \mid\left[\alpha_{2 q}\right]_{k}(z)=a_{4 q} \bar{a}_{4 q}(4 q)^{-(k-1)}\left(2^{1-k} \bar{a}_{2} f(z / 2)-f(z)\right)$. By (iii), we have $a_{4 q} \bar{a}_{4 q}=(4 q)^{k-1}$ and $a_{2} \bar{a}_{2}=2^{k-1}$. Therefore we obtain the desired result.

Proposition 1.3. Under the same conditions as in Proposition 1.1, we have $f \mid\left[\alpha_{q}\right]_{k}=i \bar{a}_{q} q^{-(k-1) / 2} f^{(q)}$, where $f^{(q)}(z)=\sum_{n=1}^{\infty} a_{n}^{(q)} e^{2 \pi i n z}$ is a primitive form with

$$
a_{p}^{(q)}= \begin{cases}\left(\frac{p}{q}\right) a_{p} & \text { if } \quad(p, q)=1 \\ \left(\frac{-4}{p}\right) \bar{a}_{p} & \text { if } \quad(p, 2)=1\end{cases}
$$

for every prime $p$.

In order to prove Proposition 1.3, we need the following lemmas.

LEMMA 1.1. $T\left(p,\left(\frac{4 q}{*}\right)\right) \alpha_{q}=\left(\frac{p}{q}\right) \alpha_{q} T\left(p,\left(\frac{4 q}{*}\right)\right)$ for every prime $p(\neq q)$ and $T\left(p,\left(\frac{4 q}{*}\right)\right) \alpha_{4}=\left(\frac{-4}{p}\right) \alpha_{4} T\left(p,\left(\frac{4 q}{*}\right)\right)$ for every prime $p(\neq 2)$.

LEMMA 1.2. Let $f(z)=\sum_{n=1}^{\infty} a_{n} e^{2 \pi \imath n z}$ be a primitive form of $S_{k}\left(\Gamma_{0}(4 q)\right.$, $\left.\left(\frac{4 q}{*}\right)\right)$. Then $f^{(q)}$ is also a primitive form of $S_{k}\left(\Gamma_{0}(4 q),\left(\frac{4 q}{*}\right)\right)$ and $f^{(q)} \mid T\left(p,\left(\frac{4 q}{*}\right)\right)=$ $a_{p}^{(q)} f^{(q)}$ for every prime $p$.

These lemmas can be proved by an argument similar to that of Asai [1] and we omit the details of the proofs.

Proof of Proposition 1.3. By Lemmas 1.1 and 1.2, we have $f \mid\left[\alpha_{q}\right]_{k}=$ $\lambda f^{(q)}$. For each $h \in \boldsymbol{Z} / q \boldsymbol{Z}(\not \equiv \mathbf{1}(\bmod q))$, we can take $j \in \boldsymbol{Z} / q \boldsymbol{Z}$ such that $j\left(1+4 h \xi^{\prime}\right) \equiv 1(\bmod q)$. Then we can see

$$
\left(\begin{array}{ll}
1 & h \\
0 & q
\end{array}\right) \alpha_{q}=\sigma_{j}\left(\begin{array}{ll}
1 & j \\
0 & q
\end{array}\right)\left(\begin{array}{ll}
q & 0 \\
0 & 1
\end{array}\right)
$$



$$
\left(\frac{4 q}{q \rho^{\prime}-4 \xi^{\prime} j}\right)=\left(\frac{j}{q}\right) \text {. }
$$

If $h \equiv 1(\bmod q)$, then $\left(\begin{array}{ll}1 & 1 \\ 0 & q\end{array}\right) \alpha_{q}=\sigma^{\prime} \alpha_{q}\left(\begin{array}{ll}q & 0 \\ 0 & 1\end{array}\right)$ with $\sigma^{\prime} \in \Gamma_{0}(4 q)$ and

$$
\boldsymbol{\sigma}^{\prime}=\left(\begin{array}{cc}
* & * \\
* & q^{2} \rho^{\prime}-4 \xi^{\prime}
\end{array}\right)
$$


so that

$$
\left(\frac{4 q}{q^{2} \rho^{\prime}-4 \xi^{\prime}}\right)=\left(\frac{-4}{q}\right) \text {. }
$$

Now, by the definition of the Hecke operator $T\left(q,\left(\frac{4 q}{*}\right)\right)$, we have

$$
\begin{aligned}
& f\left|T\left(q,\left(\frac{q q}{*}\right)\right)\right|\left[\alpha_{q}\right]_{k}=q^{k / 2-1} \sum_{h=0}^{q-1} f\left|\left[\left(\begin{array}{ll}
1 & h \\
0 & q
\end{array}\right)\right]_{k}\right|\left[\alpha_{q}\right]_{k} \\
& =q^{k / 2-1}\left\{\sum_{\substack{h=0 \\
h \neq 1}}^{q-1} f\left|\left[\left(\begin{array}{ll}
1 & h \\
0 & q
\end{array}\right) \alpha_{q}\right]_{k}+f\right|\left[\left(\begin{array}{ll}
1 & 1 \\
0 & q
\end{array}\right) \alpha_{q}\right]_{k}\right\} \\
& =q^{k / 2-1}\left\{\sum_{j=1}^{q-1} f\left|\left[\sigma_{j}\left(\begin{array}{ll}
1 & j \\
0 & q
\end{array}\right)\left(\begin{array}{ll}
q & 0 \\
0 & 1
\end{array}\right)\right]_{k}+f\right|\left[\sigma^{\prime} \alpha_{q}\left(\begin{array}{ll}
q & 0 \\
0 & 1
\end{array}\right)\right]_{k}\right\} \\
& =q^{k / 2-1} \sum_{n=1}^{\infty} a_{n}\left\{\sum_{j=1}^{q}\left(\frac{j}{q}\right) e^{2 \pi i n j / q}\right\} e^{2 \pi i n z}+q^{k-1}\left(\frac{-4}{q}\right) \lambda \sum_{n=1}^{\infty} a_{n}^{(q)} e^{2 \pi i n q z} \text {. }
\end{aligned}
$$

On the other hand, we see that

$$
f\left|T\left(q,\left(\frac{4 q}{*}\right)\right)\right|\left[\alpha_{q}\right]_{k}=a_{q} f \mid\left[\alpha_{q}\right]_{k}=a_{q} \lambda \sum_{n=1}^{\infty} a_{n}^{(q)} e^{2 \pi i n z} .
$$

Comparing two Fourier expansions, we obtain the required result.

2. Maass' theta function. We consider the real quadratic field $F=$ $\boldsymbol{Q}(\sqrt{4 q})$ of class number one. Let $\xi_{m}(m \in \boldsymbol{Z})$ be a Grössen character of $F$ defined by $\xi_{m}(\mathfrak{a})=\left|\alpha / \alpha^{\prime}\right|^{2 m \pi / \log \varepsilon_{0}}$ for an ideal $\mathfrak{a}=(\alpha)$, where $\varepsilon_{0}(>1)$ is the fundamental unit in $F$. Let $g\left(z ; \xi_{m}\right)$ be a real analytic automorphic function attached to the $L$-function of $F$, that is,

$$
g\left(z ; \xi_{m}\right)=C_{\xi_{m}} y^{1 / 2}+y^{1 / 2} \sum_{\mathfrak{a} \neq 0} \xi_{m}(\mathfrak{a}) K_{\imath m \pi / \log \varepsilon_{0}}(2 \pi N(\mathfrak{a}) y) \times\left(e^{-2 \pi i N(\mathfrak{a}) x}+e^{2 \pi i N(\mathfrak{a}) x}\right),
$$

where $z=x+i y \in \mathfrak{S}$. It is well-known (Maass [6]) that $g\left(z ; \xi_{m}\right)$ has the following properties:

(1) $g\left(z ; \xi_{m}\right)=0\left(y^{\delta}\right)\left(\right.$ resp. $\left.0\left(y^{-\delta^{\prime}}\right)\right)$ uniformly in $x$, as $y \rightarrow \infty($ resp. $y \rightarrow 0)$, where $\delta, \delta^{\prime}>0$,

(2) $g\left(\gamma(z) ; \xi_{m}\right)=\left(\frac{4 q}{d}\right) g\left(z ; \xi_{m}\right)$ for each

$$
\gamma=\left(\begin{array}{ll}
* & * \\
* & d
\end{array}\right) \in \Gamma_{0}(4 q),
$$

and

$$
g\left(\alpha_{4 q}(z) ; \xi_{m}\right)=g\left(z ; \xi_{m}\right) .
$$

Let $M$ be a positive integer with $M \mid 4 q$ and $M \neq 2,2 q$ and define the function $g^{(M)}\left(z ; \xi_{m}\right)$ by 


$$
\begin{aligned}
g^{(M)}\left(z ; \xi_{m}\right)= & \varepsilon_{M}\left(C_{\xi_{m}}^{(M)} y^{1 / 2}+y^{1 / 2} \sum_{\mathfrak{a} \neq 0} \psi_{M}(\mathfrak{a}) \xi_{m}(\mathfrak{a}) K_{\imath m \pi / \log \varepsilon_{0}}(2 \pi N(\mathfrak{a}) y)\right. \\
& \left.\times\left(e^{2 \pi i N(\mathfrak{a}) x}+\delta_{M} e^{-2 \pi i N(\mathfrak{a}) x}\right)\right)
\end{aligned}
$$

where

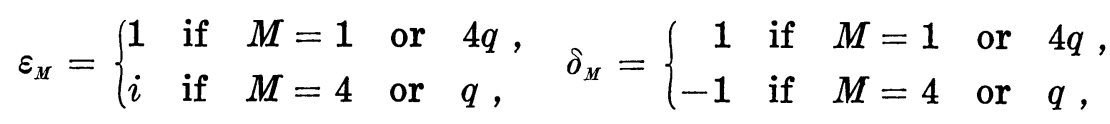

$\psi_{M}(\mathfrak{p})=1$ if $M=1$ or $4 q$ and

$$
\psi_{M}(\mathfrak{p})=\left\{\begin{array}{lll}
\left(\frac{-4}{N(\mathfrak{p})}\right) & \text { if } & \mathfrak{p} \nmid 2 \\
\left(\frac{N(\mathfrak{p})}{p}\right) & \text { if } & \mathfrak{p} \nmid q
\end{array}\right.
$$

if $M=4$ and $q$ for each prime ideal $\mathfrak{p}$.

By an argument similar to that in $\S 1$, we can obtain the following:

Proposition 2.

(1) $g\left(\alpha_{M}(z) ; \xi_{m}\right)=g^{(M)}\left(z ; \xi_{m}\right)$ if $M=1,4, q$ or $4 q$,

(2) $g\left(\alpha_{2 q}(z) ; \xi_{m}\right)=\sqrt{2} \xi_{m}\left(\mathfrak{p}_{0}\right) g\left(z / 2 ; \xi_{m}\right)-g\left(z ; \xi_{m}\right)$,

where $\mathfrak{p}_{0}^{2}=(2)$.

3. Eisenstein-Epstein functions. We define $S^{\times}$and $S$ as follows: $S^{\times}=\left\{(\alpha, \beta) \in Z^{2} \mid(\alpha, \beta)=1, \alpha \beta \neq 0\right\}$ and $S=S^{\times} \cup\{( \pm 1,0)\} \cup\{(0, \pm 1)\}$. Let $k$ be an even posititive integer and $M$ a positive integer with $M \mid 4 q$. Put

$$
E_{M}(z, s, k)_{4 q}=(1 / 2) y^{8} \sum_{\substack{(\mu, \nu) \in S \\(\mu, 4 q)|=4 q| M}}(\mu z+\nu)^{k}|\mu z+\nu|^{-(2 s+k)},
$$

where $z=x+i y \in \mathfrak{S}$ and $s \in C$. The series on the right hand side is absolutely convergent for $\operatorname{Re} s>1$, and moreover it can be continued analytically to the whole $s$-plane. We put

$$
E_{M}(z, s, k)_{4 q} \mid[\alpha]_{k}=((c z+d) /|c z+d|)^{k} E_{M}(\alpha(z), s, k)_{4 q}
$$

for all

$$
\alpha=\left(\begin{array}{ll}
a & b \\
c & d
\end{array}\right) \in G L_{2}^{+}(\boldsymbol{R})
$$

We can prove the following lemma.

LEMMA 3.1 .

(1) $E_{2}(z, s, k)_{4 q}=E_{1}(z, s, k)_{4 q} \mid\left[\alpha_{2 q}\right]_{k}$, 


$$
\begin{aligned}
& E_{\mu k}(z, s, k)_{s q}=M^{s} E_{1}(z, s, k)_{4 q} \mid\left[\alpha_{\mu}\right]_{k} \text { if } M=1,4, q \text { or } 4 q, \\
& \text { (2) } E_{2}(z, s, k)_{4 q} \mid\left[\alpha_{4 q}\right]_{k}=q^{-8} E_{2 q}(z, s, k)_{4 q} .
\end{aligned}
$$

Proof. We shall prove (2). We put

$$
S_{M}^{4 q}=\{(\mu, \nu) \in S||(\mu, 4 q) \mid=4 q / M\} .
$$

Consider the isomorphism $\phi: S_{2}^{4 q} \rightarrow S_{2 q}^{4 q}$ such that $\phi(\mu, \nu)=(2 \nu,-(\mu / 2 q))$. Then we have

$$
\begin{aligned}
E_{2}(z, & s, k)_{4 q} \mid\left[\alpha_{4 q}\right]_{k} \\
& =(1 / 2)(z /|z|)^{k}\left(y / 4 q|z|^{2}\right)^{s} \sum_{(\mu, \nu) \in S_{2 q}^{4 q}}(\mu(-1 / 4 q z)+\nu)^{k}|\mu(-1 / 4 q z)+\nu|^{-(2 s+k)} \\
& =(1 / 2)(z /|z|)^{k}\left(y / 4 q|z|^{2}\right)^{s} \sum_{\left(\mu^{\prime}, \nu^{\prime}\right) \in S_{2 q}^{4 q}}\left(\left(\mu^{\prime} z+\nu^{\prime}\right) / 2 z\right)^{k}\left|\left(\mu^{\prime} z+\nu^{\prime}\right) / 2 z\right|^{-(2 s+k)} \\
& =q^{-s} E_{2 q}(z, s, k)_{4 q},
\end{aligned}
$$

which completes the proof. Since we can prove (1) by an argument similar to the proof of (2), we omit the details of the proof. In order to get the functional equation of $\left\{E_{M}(z, s, k)_{4 q}\right\}_{M \mid q q}$, we define another function $E^{*}(z, s, k)$ by

$$
E^{*}(z, s, k)=(1 / 2) \pi^{-s} \Gamma(s+k / 2) \zeta(2 s) y_{(\mu, \nu) \in S}^{s}(\mu z+\nu)^{k}|\mu z+\nu|^{-(28+k)},
$$

where $\zeta(s)$ is the Riemann zeta function. We have the following (cf. Shimura [7]).

LEMma 3.2. The function $E^{*}(z, s, k)=\pi^{-s} \Gamma(s+k / 2) \zeta(2 s) \times \sum_{M \mid 4 q} E_{M}(z$, $s, k)_{4 q}$ can be continued to the whole s-plane as an entire function satisfying the functional equation

$$
E^{*}(z, s, k)=E^{*}(z, 1-s, k) .
$$

4. Rankin's method and the Doi-Naganuma lifting. Let $f(z)=$ $\sum_{n=1}^{\infty} a(n) e^{2 \pi i n z}$ be a primitive form in $S_{k}\left(\Gamma_{0}(4 q),\left(\frac{4 q}{*}\right)\right)$ and put

$$
\left(f \mid\left[\alpha_{M}\right]_{k}\right)(z)=\sum_{n=1}^{\infty} a_{M}(n) e^{2 \pi i n z} / c_{M}, \quad \text { where } c_{M}=\left\{\begin{array}{lll}
1 & \text { if } M=1,4, q \text { or } 4 q, \\
2 & \text { if } M=2 q \text { or } 2 .
\end{array}\right.
$$

For each integral ideal $a$ of $F$, we define $C_{a}$ in the following manner: For a prime ideal $\mathfrak{p}$ in $F$, we put

$$
\begin{aligned}
& C_{\mathfrak{p}}=C_{\mathfrak{p}^{\prime}}=a(p) \quad \text { if } \quad \mathfrak{p p}^{\prime}=(p) \quad \text { and } \quad \mathfrak{p} \neq \mathfrak{p}^{\prime} \text {, } \\
& C_{\mathfrak{p}}=a(p)^{2}+2 p^{k-1} \quad \text { if } \mathfrak{p}=(p) \text { ， } \\
& C_{\mathfrak{p}}=a(p)+\overline{a(p)} \quad \text { if } \quad \mathfrak{p}^{2}=(p) \text { ， }
\end{aligned}
$$

and define 


$$
\begin{aligned}
C_{p e} & =C_{\mathfrak{p}} \cdot C_{p} e-1-N(\mathfrak{p})^{k-1} C_{\mathfrak{p} e-2} \quad(e \geqq 2), \\
C_{\mathfrak{a}} & =\prod_{i} C_{p_{i}^{e i}} \quad \text { if } \quad \mathfrak{a}=\prod_{\mathfrak{i}} \mathfrak{p}_{i}^{e i} .
\end{aligned}
$$

Put $D\left(s ; \xi_{m}\right)=\sum_{\mathfrak{a}} \xi_{m}(\mathfrak{a}) C_{\mathfrak{a}} N(\mathfrak{a})^{-s}$ and

$$
D^{*}\left(s ; \xi_{m}\right)=(2 \pi)^{-2 s}(4 q)^{8} \Gamma\left(s+i m \pi / \log \varepsilon_{0}\right) \Gamma\left(s-i m \pi / \log \varepsilon_{0}\right) D\left(s ; \xi_{m}\right) .
$$

We can prove the following theorem.

THEOREM. In the above notations, $D^{*}\left(s ; \xi_{m}\right)$ can be continued to the whole s-plane as an entire function and it satisfies the functional equation $D^{*}\left(s ; \xi_{m}\right)=D^{*}\left(k-s ; \xi_{m}\right)$. Furthermore,

$$
D\left(s ; \xi_{0}\right)=\sum_{n=1}^{\infty} a(n) n^{-s} \sum_{n=1}^{\infty} \overline{a(n)} n^{-s} \text {. }
$$

Proof. We put

$$
D^{*}\left(s^{\prime}\right)=\int_{D_{0}(4 q)} y^{k / 2} f(z) \overline{g\left(z ; \xi_{m}\right)} E^{*}\left(z, s^{\prime}, k\right) y^{-2} d x d y,
$$

where $D_{0}(4 q)$ denotes a fundamental region for $\Gamma_{0}(4 q)$. Then we can isee $(* * *)$

$$
D^{*}\left(s^{\prime}\right)=\pi^{k / 2} D^{*}\left(s ; \xi_{m}\right), \quad\left(s=s^{\prime}+(k-1) / 2\right) .
$$

In fact, we have

$$
D^{*}\left(s^{\prime}\right)=(4 q)^{s^{\prime}} \sum_{M i 4 q} I_{M}\left(s^{\prime}\right)
$$

Here

$$
I_{M}\left(s^{\prime}\right)=(4 q)^{-s^{\prime}} \pi^{-s^{\prime}} \Gamma\left(s^{\prime}+k / 2\right) \zeta\left(2 s^{\prime}\right) \int_{D_{0}(4 q)} y^{k / 2} f(z) \overline{g\left(z ; \xi_{m}\right)} E_{M}\left(z, s^{\prime}, k\right)_{4 q} y^{-2} d x d y .
$$

First, we compute $I_{2}\left(s^{\prime}\right)$. By Lemma $3.1(1)$, we have

$$
\begin{aligned}
I_{2}\left(s^{\prime}\right)= & \left.(4 q)^{-s^{\prime}} \zeta\left(2 s^{\prime}\right) \pi^{-s^{\prime}} \Gamma\left(s^{\prime}+k / 2\right) \int_{D_{0}(4 q)} y^{k / 2} f(z) \overline{g\left(z ; \xi_{m}\right.}\right) \\
& \times E_{1}\left(z, s^{\prime}, k\right)_{4 q} \mid\left[\alpha_{2 q}\right]_{k} y^{-2} d x d y \\
= & (4 q)^{-s^{\prime}} \zeta\left(2 s^{\prime}\right) \pi^{-s^{\prime}} \Gamma\left(s^{\prime}+k / 2\right) \int_{\alpha_{2 q}\left(D_{0}(4 q)\right)} y^{k / 2} f(z) \\
& \left.\times \overline{g\left(z ; \xi_{m}\right.}\right) \mid\left[\alpha_{2 q}^{-1}\right]_{k} \times E_{1}\left(z, s^{\prime}, k\right)_{4 q} y^{-2} d x d y .
\end{aligned}
$$

Since $\alpha_{2 q}$ normalizes $\Gamma_{0}(4 q)$, we have

$$
\begin{aligned}
I_{2}\left(s^{\prime}\right)= & \left.(4 q)^{-s^{\prime}} \zeta\left(2 s^{\prime}\right) \pi^{-s^{\prime}} \Gamma\left(s^{\prime}+k / 2\right) \int_{D_{0}(4 q)} y^{k / 2} f \mid\left[\alpha_{2 q}^{-1}\right]_{k} \overline{g\left(\alpha_{2 q}^{-1}(z) ; \xi_{m}\right.}\right) \\
& \times E_{1}\left(z, s^{\prime}, k\right)_{4 q} y^{-2} d x d y \\
= & \left.(4 q)^{-s^{\prime}} \zeta\left(2 s^{\prime}\right) \pi^{-s^{\prime}} \Gamma\left(s^{\prime}+k / 2\right) \int_{0}^{\infty} \int_{0}^{1} f \mid\left[\alpha_{2 q}^{-1}\right]_{k} \overline{g\left(\alpha_{2 q}^{-1}(z) ; \xi_{m}\right.}\right) \\
& \times y^{s^{\prime}+(k / 2)-2} d x d y
\end{aligned}
$$


By Proposition 2, we have the following (cf. [3]):

$$
\begin{aligned}
I_{2}\left(s^{\prime}\right)= & \pi^{k / 2}(4 q)^{(k-1) / 2}(2 \pi)^{-2 s} \Gamma\left(s+i m \pi / \log \varepsilon_{0}\right) \Gamma\left(s-i m \pi / \log \varepsilon_{0}\right) q^{-8} 2^{-s} \\
& \times \xi_{m}\left(\mathfrak{p}_{0}\right) \zeta(2 s-k+1) \sum_{n=1}^{\infty} a_{2 q}(n)\left(\sum_{N(\mathfrak{a})=n} \xi_{m}(\mathfrak{a})\right) n^{-s} .
\end{aligned}
$$

Next, by virtue of Lemma 3.1 (2), we see that

$$
\begin{aligned}
I_{2 q}\left(s^{\prime}\right)= & \left.4^{-s^{\prime} \zeta}\left(2 s^{\prime}\right) \pi^{-s} \Gamma\left(s^{\prime}+k / 2\right) \int_{D_{0}(4 q)} y^{k / 2} f(z) \overline{g\left(z ; \xi_{m}\right.}\right) \\
& \times E_{2}\left(z, s^{\prime}, k\right)_{4 q} \mid\left[\alpha_{4 q}\right]_{k} y^{-2} d x d y \\
= & 4^{-s^{\prime}} \zeta\left(2 s^{\prime}\right) \pi^{-s^{\prime}} \Gamma\left(s^{\prime}+k / 2\right) \int_{\alpha_{4 q}\left(D_{0}(4 q)\right)} y^{k / 2} f(z) \overline{\left.g(z) ; \xi_{m}\right)} \mid\left[\alpha_{4 q}^{-1}\right]_{k} \\
& \times E_{2}\left(z, s^{\prime}, k\right)_{4 q} y^{-2} d x d y \\
= & \pi^{k / 2} 4^{(k-1) / 2}(2 \pi)^{-28} \Gamma\left(s+i m \pi / \log \varepsilon_{0}\right) \Gamma\left(s-i m \pi / \log \varepsilon_{0}\right) 2^{-s} \xi_{m}\left(\mathfrak{p}_{0}\right) \\
& \times \zeta(2 s-k+1) \sum_{n: \text { odd }} a_{2}(n)\left(\sum_{N(a)=n} \xi_{m}(\mathfrak{a})\right) n^{-s} .
\end{aligned}
$$

By a similar argument, we obtain

$$
\begin{aligned}
I_{M}(s)= & \pi^{k / 2}(4 q / M)^{(k-1) / 2}(2 \pi)^{-2 s} \Gamma\left(s+i m \pi / \log \varepsilon_{0}\right) \Gamma\left(s-i m \pi / \log \varepsilon_{0}\right) \varepsilon_{M}^{-1} \\
& \times(4 q / M)^{-s} \zeta(2 s-k+1) \sum_{n=1}^{\infty} a_{M}(n)\left(\sum_{N(\mathfrak{a})=n} \xi_{m}(\mathfrak{a}) \psi_{M}(\mathfrak{a})\right) n^{-s}
\end{aligned}
$$

for $M=1,4, q$ or $4 q$. Moreover, by the propositions in $\S 1$, we have $(* * *)$ and we are done. We omit the details of the remainder of the argument.

As a corollary we have

CoRollary. Suppose that $f$ is a primitive form in $S_{k}\left(\Gamma_{0}(4 q),\left(\frac{4 q}{*}\right)\right)$. Then

$$
\sum_{n=1}^{\infty} a(n) n^{-s} \sum_{n=1}^{\infty} \overline{a(n)} n^{-s}
$$

is the Dirichlet series associated with a Hilbert modular cusp form of weight $k$ with respect to $G L_{2}\left(\mathfrak{O}_{F}\right)$.

\section{REFERENCES}

[1] T. AsAI, On the Fourier coefficients of automorphic forms at various cusps and some applications to Rankin's convolution, J. Math. Soc. Japan, 28 (1976), 48-61.

[2] Atrin-Lehner, Hecke operators on $\Gamma_{0}(m)$, Math. Ann., 185 (1970), 134-160.

[3] K. Doi and H. Naganuma, On the functional equation of certain Dirichlet series, Invent. Math., 9 (1969), 1-14.

[4] K. DoI AND T. Miyake, Automorphic forms and number theory (Japanese), Kinokuniya Shoten, Tokyo, 1976. 
[5] H. Naganuma, On the coincidence of two Dirichlet series associated with cusp forms of Hecke's Neben-type and Hilbert modular forms over a real quadratic field, J. Math. Soc. Japan, 25 (1973), 547-555.

[6] H. MaASs, Uber eine neue Art von nichtanalytischen automorphen Funktionen und die Bestimmung Dirichletscher Reihen durch Funktionalgleichungen, Math. Ann., 121 (1949), 141-183.

[7] G. SHIMURA, On modular forms of half integral weight, Ann. of Math., 97 (1973), 440481.

[8] D. ZAGIER, Modular forms associated to real quadratic fields, Invent. Math., 30 (1975), 1-46.

Mathematical INSTitute

TÔHOKU UNIVERSITY

SENDAI, 980 JAPAN 
